\title{
Práticas educativas de genitores e professores e repertório comportamental infantil
}

\section{Educational practices of parents and teachers and children's behavioral repertoire}

\author{
Bárbara Cristina Rodrigues Fonseca(orcid.org/0000-0002-9459-1053) ${ }^{1}$ \\ Alessandra Turini Bolsoni-Silva (orcid.org/0000-0002-0286-780X) ${ }^{2}$ \\ Ligia Melchiori Ebner (orcid.org/0000-0002-4771-8832) ${ }^{3}$
}

\begin{abstract}
Resumo
O objetivo deste estudo é descrever, a partir de múltiplos instrumentos e informantes, o repertório comportamental (habilidades sociais e problemas de comportamento) de quatro crianças de 6 a 7 anos, duas de cada sexo, e as práticas educativas positivas (habilidades sociais educativas) e negativas de quatro mães, quatro pais e duas professoras dos primeiros anos do Ensino Fundamental. Foram utilizados na coleta de dados instrumentos de relato e protocolo de observação de interações genitores-criança a partir de situação de brincadeira. Os resultados reafirmam que práticas positivas estão relacionadas à baixa frequência de problemas de comportamento e presença de habilidades sociais e, ao contrário, práticas negativas estão relacionadas a problemas de comportamento. A literatura tem encontrado tais relações entre pais e filhos e com a presente pesquisa nota-se o mesmo no que se refere à interação professor-aluno.
\end{abstract}

Palavras-chave: Habilidades sociais educativas. Práticas negativas. Problemas de comportamento. Habilidades sociais.

\footnotetext{
Abstract

The aim of this study is to describe, from multiple instruments and informants, the behavioral repertoire (social educational practices (educational social skills) and negative practices of four mothers, four fathers and two teachers from the first years of elementary school. Data collection instruments and protocol for observing the parent-child

1 Universidade Estadual Paulista, São Paulo, Brasil. E-mail: babi.fonseca2121@hotmail.com.

2 Universidade Estadual Paulista, São Paulo, Brasil. E-mail: bolsonisilva@gmail.com.

3 Universidade Estadual Paulista, São Paulo, Brasil. E-mail: ligia.melch@gmail.com.
} skills and behavioral problems) of four children aged six to seven years old, two of each sex, and positive 
interactions, from a situation of play, were used. The results reaffirm that positive practices are related to the low frequency of behavioral problems and the presence of social skills, and, on the contrary, negative practices are related to behavioral problems. The literature has found such relationships between parents and children and with the present research the same can be noticed with regard to teacher-student interaction.

Keywords: Educational social skills. Negative practices. Behavioral problems. Social skills.

O estudo das interações sociais na família e na escola, entre vários aspectos, justifica-se porque pais e professores podem avaliar diferentemente os comportamentos sociais das crianças (Rescorla et al., 2014; Rudasill, et al., 2014) em ambientes distintos, pois as crianças podem emitir respostas diferentes de acordo com demandas e exigências próprias do contexto, podendo haver crianças que tenham problemas apenas em um deles ou nos dois. Adicionalmente, o repertório de habilidades sociais e de problemas de comportamento infantil parecem ser multideterminados, em que diferentes variáveis os influenciam, tais como práticas educativas negativas e positivas (pais e professores), psicopatologia parental, variáveis sociodemográficas, sexo e escolaridade da criança (Lins, Alvarenga, Santos, Almeida \& Santos, 2012).

Pesquisas têm encontrado associações entre práticas educativas positivas e habilidades sociais infantis (Bolsoni-Silva, Loureiro \& Marturano, 2016; Borden et al., 2014; Marin, Piccinini, Gonçalves \& Tudge, 2012), bem como evidências da presença de mais habilidades sociais em grupos não clínicos do que nos grupos julgados clínicos para problemas de comportamento (Barreto, Freitas \& Del Prette, 2011 ; Bolsoni-Silva \& Loureiro, 2011). Por outro lado, pesquisadores também têm encontrado relação entre práticas educativas negativas e problemas de comportamento (Alvarenga, Magalhães \& Gomes, 2012; Borden et al., 2014; Bolsoni-Silva \& Loureiro, 2011; Shaffer, Lindhiem, Kolko \& Trentacosta, 2013; Xing \& Wang, 2013).

A literatura da psicopatologia infantil caracteriza como problemas de comportamento os externalizantes e os internalizantes. Os externalizantes são descritos com termos como agressividade, desobediência e comportamentos antissociais (Casali- 
Robalinho, Del Prette \& Del Prette, 2015). Geralmente são esses problemas externalizantes que causam mais incômodo aos pais e professores, pois podem ser observados diretamente e demonstram pouco autocontrole e autorregulação da criança (Merrell \& Harlacher, 2008), compondo, consequentemente, a maior parte das queixas sobre o comportamento infantil (Bolsoni-Silva, Levatti, Guidugli \& Marim, 2015; Pesce, 2009). Os comportamentos chamados internalizantes são comumente referidos como timidez, medo, problemas gastrointestinais, recusa de ir à escola e tristeza (Lins \& Alvarenga, 2015).

Enquanto taxonomia de comportamentos de habilidades sociais, Bolsoni-Silva, Marturano, Figueiredo e Manfrinato, (2006) propõem três amplas categorias comportamentais para as habilidades sociais infantis: a) disponibilidade social e cooperação; b) expressão de sentimentos e enfrentamento; c) interação social positiva. Tais comportamentos diferenciaram crianças que obtiveram classificações clínica ou não clínica para problemas de comportamento, seja por meio do relato de mães, seja por meio de relato de professores (Bolsoni-Silva \& Loureiro, 2016). As práticas parentais têm sido referenciadas de diferentes formas na literatura em sua relação com problemas de comportamento: a) excesso de monitoria negativa (Gomide, 2006; Salvo, Silvares \& Toni, 2005); b) agressividade (Price, Chiapa \& Walsh, 2013; Trepat, Granero \& Ezpeleta, 2014; Xing \& Wang, 2013); c) deficit de supervisão ou monitoria positiva (Gomide, 2006; Salvo et al., 2005); d) deficit em comunicação (Bolsoni-Silva \& Mariano, 2014; Borden et al., 2014; Bueno, Grossi, Santo, Silva \& Moura, 2011); e) deficit de afeto (Bolsoni-Silva \& Loureiro, 2011 ; O’Connor, Dearing \& Collins, 2011; Silver, Measelle, Armstrong \& Essex, 2010); f) inconsistência no estabelecimento de limites (Leme \& Bolsoni-Silva, 2010; Marin et al., 2012).

Caracterizam-se, na presente pesquisa, as práticas positivas de educação como Habilidades Sociais Educativas (HSE) de pais (Bolsoni-Silva et al., 2016) e de professores (Mariano, 2011), sendo elas descritas em três grandes categorias: a) comunicação, b) expressão de sentimentos e enfrentamento; e c) estabelecimento de limites. Ainda que práticas parentais e HSE tenham conceitos diversos na literatura, Sabbag e Bolsoni-Silva 
(2015), de maneira empírica, coletaram dados em uma cidade de médio porte do estado do Paraná, utilizando o Roteiro de Entrevista de Habilidades Sociais Educativas Parentais - REHSE-P (Bolsoni-Silva et al., 2016) e o Inventário de Estilo Parental - IEP (Gomide, 2006), e encontraram correlações positivas entre monitoria positiva e comportamento moral com as habilidades sociais educativas e, adicionalmente, as habilidades sociais educativas no REHSE-P foram inversamente proporcionais às práticas negativas no IEP, bem como houve a convergência da avaliação de práticas negativas pelos dois instrumentos. Desse modo, é possível verificar consonância entre prática parental e habilidades sociais educativas.

No que se refere à interação professor-aluno, as pesquisas (Maia et al., 2010; Mariano, 2015; Reis, Prata \& Soares, 2012; Soares \& Mello, 2010), embora mais escassas na comparação das interações dos pais com os filhos, de modo geral, têm encontrado resultados na mesma direção, ressaltando a importância das práticas positivas do professor na promoção de habilidades sociais e na redução de problemas de comportamento, abrangendo a comunicação, o afeto e o estabelecimento de limites. A título de exemplo, ao investigar práticas educativas e comportamentos infantis de crianças de seis anos de idade, Bolsoni-Silva e Mariano (2014) verificaram que as professoras apresentavam práticas positivas de interação, sobretudo as de comunicação. No entanto, a qualidade da comunicação era diferente conforme identificavam as crianças com e sem problemas de comportamento. Com as crianças julgadas clínicas, as professoras conversavam de forma mais contingente a comportamentos considerados como problemas, sobre concepções de certo e errado, e não a temas diversos ou pessoais, como ocorria com as crianças julgadas não clínicas, as quais também recebiam mais atenção e afeto; tais achados podem ser explicados, considerando que crianças com comportamentos identificados como clínicos são mais difíceis de lidar e os adultos tendem a usar mais controle aversivo para regular o comportamento e pouco utilizam de estratégias positivas (Patterson, Reid \& Dishion, 2002).

Quanto a múltiplos avaliadores para mensurar comportamentos infantis, é possível encontrar diversos estudos, mas com resultados pouco conclusivos, pois alguns estudos verificaram que mães e professores avaliam de maneira igual (Bernedo, Salas, Fuentes \& 
Garcia-Martin, 2014; Ercan, Bilaç, Özazlan \& Rohde, 2014, entre outros) e diferente os comportamentos (Korsch \& Petermann, 2013; Lavigne, Dahl, Lebailly \& Hopkins, 2014; Rescorla et al., 2014; Rudasill et al., 2014). Além desse aspecto, até onde pode alcançar esta revisão, as avaliações são via relato, não incluindo observação das interações adultoscriança. Outro aspecto a considerar é que, para a maioria dos estudos, os comportamentos das crianças e as práticas educativas dos pais e professores são estudados de maneira separada, sendo que poucos buscaram avaliar concomitantemente esses comportamentos, ou seja, as interações sociais estabelecidas entre pais e crianças e entre professores e alunos em uma mesma pesquisa.

O objetivo deste estudo é descrever, a partir de múltiplos instrumentos e informantes, o repertório comportamental (habilidades sociais e problemas de comportamento) de quatro crianças de 6 a 7 anos, duas de cada sexo, e as práticas educativas positivas (habilidades sociais educativas) e negativas de quatro mães, quatro pais e duas professoras dos primeiros anos do Ensino Fundamental.

\section{Método}

\section{Participantes}

Este estudo contou com 14 participantes, sendo duas professoras (uma de $1^{\circ}$ e outra do $2^{\circ}$ ano) de uma Escola Municipal de Ensino Fundamental, quatro escolares e seus genitores (quatro pais e quatro mães). As professoras tinham entre 43 e 47 anos de idade, de 20 a 25 anos de profissão e renda familiar de quatro a seis salários mínimos. Ambas tinham curso Superior Completo, com especialização em Psicopedagogia. Por critérios de inclusão dos pais, eles precisavam conviver maritalmente e foram consideradas crianças de ambos os sexos, sendo parte com indicadores de problemas de comportamento e parte sem problemas, avaliadas pelas professoras no contexto escolar. O percurso amostral encontrase descrito em procedimentos de coleta de dados. 
Em relação às crianças, duas eram do sexo masculino (André, sete anos e Caio, sete anos e cinco meses) e duas do feminino (Beatriz, seis anos e oito meses e Daiane, sete anos). ${ }^{4}$ As crianças com iniciais A e B não apresentaram indicadores de problemas de comportamento, e as com iniciais C e D apresentaram esses problemas. Todos os genitores eram biológicos, legalmente casados há mais de 10 anos. As mães tinham entre 29 e 38 anos; destas, uma havia concluído o ensino médio completo, as outras haviam concluído o Ensino Fundamental. Os pais tinham entre 29 e 35 anos e três haviam concluído o Ensino Fundamental, sendo que um deles o fez de forma incompleta. Mães e pais com ocupações remuneradas exerciam atividades diversificadas e tinham rendimentos variados, com renda per capta de menos da metade de um salário mínimo a um salário. Uma mãe não exercia atividade remunerada.

\section{Instrumentos e materiais}

Roteiro de Entrevista de Habilidades Sociais Educativas Parentais - RE-HSE-P (Bolsoni-Silva et al., 2016) e Roteiro de Entrevista de Habilidades Sociais Educativas para Professores - RE-HSE-Pr (Bolsoni-Silva, Marturano \& Loureiro, 2018), que avaliam a diversidade (variabilidade com que os comportamentos ocorrem, por exemplo, se o respondente relata que para estabelecer limites vale-se do carinho, da conversa, do estabelecimento de regras; nesse caso teria o escore 3 para habilidades sociais educativas de estabelecer limites) e a frequência dos comportamentos - refere-se, por exemplo, a perguntar com que frequência na semana o respondente utiliza das estratégias mencionadas para estabelecer limites, que podem ser nunca/quase nunca (escore 0), algumas vezes (escore 1) ou frequentemente (escore 2). Nos instrumentos há instruções para codificar as frequências com escores de 0 a 2 . Os construtos gerais avaliados são habilidades sociais educativas (práticas positivas), práticas educativas negativas, comportamentos das crianças de habilidades sociais e de problemas de comportamento. As

\footnotetext{
${ }^{4}$ Todos os nomes das crianças são fictícios.
} 
subcategorias utilizadas estão descritas no Protocolo de Observação. Os instrumentos são entrevistas conduzidas pelo psicólogo, que faz perguntas previstas sobre as interações sociais estabelecidas entre pais e filhos e entre professores e alunos. As entrevistas são gravadas e posteriormente codificadas de acordo com os Manuais dos referidos instrumentos. Os testes têm aprovações do Conselho Federal de Psicologia e são de uso exclusivo de psicólogos. Ambos, na validação de construto, apresentaram dois fatores: Total Positivo (habilidades sociais educativas e habilidades sociais) e Total Negativo (práticas negativas e problemas de comportamento). Podem ser aplicados com crianças a partir de três até 12 anos. Quanto à consistência interna, a versão para pais apresentou um alpha de 0,846 e a de professor o alfa foi de 0,700. A partir de Curvas Roc, conduzidas com a versão para pais, identificaram-se pontos de corte para os construtos mensurados nos instrumentos, que permitem classificá-los como clínicos (C), limítrofes (L) e não clínicos (NC). Os testes conseguiram, nas validades discriminantes, diferenciar meninos de meninas, escolares de pré-escolares.

CBCL "Child Behavior Checklist" e TRF "Teacher's Report Form" (Inventários de Comportamentos da Infância e Adolescência, Achenbach \& Rescorla, 2001) avaliam indicadores de problemas de comportamento, competência social e acadêmica do ensino infantil ao fundamental (1,5 a 5 anos e 6 a 18 anos), considerando os relatos de pais (CBCL) ou de professores (TRF). Os respondentes, em escala tipo likert, assinalam a frequência de 113 ou 118 (a depender da faixa etária) de respostas indicativas de problemas de comportamento por meio dos escores "não é verdadeiro $=0$ ", "um pouco verdadeiro $=1$ " e "muito verdadeiro $=2$ ", e de perguntas relacionadas à vida escolar, social e familiar da criança. Os resultados podem ser organizados em problemas internalizantes, externalizantes e totais, que podem ser classificados como clínicos, limítrofes e não clínicos por meio de análise computadorizada (Software ADM - 2006). O CBCL tem validação preliminar e ambos os instrumentos estão em processos de validação no Brasil, os quais são considerados mundialmente como padrão ouro para avaliar problemas de comportamento (Bordin, et al., 2013). 
O Protocolo de Observação foi elaborado a partir de categorias extraídas do instrumento RE-HSE-P (Bolsoni-Silva et al, 2016), por ser um instrumento validado para essa população. A ocasião da observação foi a da aplicação de jogo de memória de 72 peças, após a viabilidade confirmada em estudo piloto. São descritas as subcategorias e categorias para os construtos avaliados a seguir.

Quanto às HSE de Comunicação e negociação, os comportamentos codificados foram: diz "não" com explicação, dá explicações, elogia sobre consequências comportamento, estabelece regras, fala/conversa, faz perguntas, pede mudança de comportamento. Quanto às HSE de Expressão de sentimentos e enfrentamento, os comportamentos são: agrada (abraça, beija, faz carinhos), brinca (oralmente), compra coisas que o filho quer, incentiva, olha para a criança, sorri, fica contente. As práticas negativas são, por exemplo, gritar, bater, xingar, fazer careta.

Quanto às habilidades sociais infantis, são organizadas em Disponibilidade Social e Cooperação: atende a pedidos, conversa com a mãe/pai, faz perguntas, oferece apoio, responde perguntas, obedece a ordens dadas, é atencioso, fala coisas engraçadas, muda comportamentos conforme solicitado, ouve o que adultos têm a dizer, aceita hábitos da família, é educado, aceita opiniões dos adultos. Outra categoria de habilidades sociais é a Expressão de Sentimentos e Enfrentamento: agrada, brinca (oralmente), demonstra contentamento, expressa opiniões, sorri, olha para pai/mãe, aceita opiniões dos adultos, negocia, pensa, tira dúvidas, respeita, demonstra contentamento, agradece, fica com a família, reivindica seus direitos, retribui afeto recebido, diz que gosta dos genitores, é alegre.

No que tange aos problemas de comportamento, podem ser externalizantes e internalizantes. Os externalizantes avaliados são: desobedece a ordens ou regras, é teimoso, insistente, faz birras, emburra, responde aos genitores e/ou pessoas mais velhas, reclama, grita, briga, xinga. E os internalizantes são: chora, fica triste, fica quieto, sente dores somáticas, sai da situação, tenta se esconder. 


\section{Procedimentos de coleta de dados}

Após a aprovação do projeto de pesquisa pelo Comitê de Ética responsável e obtida a anuência da Secretaria de Ensino, a pesquisadora entrou em contato com a diretora e professoras dos $1^{\circ}$ e $2^{\circ}$ anos de uma Escola Municipal de Ensino Fundamental, escolhida por conveniência. Dadas as explicações do objetivo da pesquisa, as professoras assinaram o Termo de Consentimento Livre e Esclarecido (TCLE). O próximo passo foi o de selecionar as crianças, a partir das respostas da professora ao TRF, que, na sequência responderam ao RE-HSE-Pr. As professoras avaliaram todas as crianças das duas salas selecionadas, num total de 60 (30 por sala); no entanto, apenas oito delas foram classificadas como apresentando problemas de comportamento nessas séries. As oito famílias de crianças avaliadas na categoria clínicas foram procuradas, mas somente em duas os genitores conviviam maritalmente e concordaram em participar, depois das devidas explicações e a assinatura do TCLE. A seguir, procurou-se o mesmo número de famílias participantes das crianças avaliadas como não clínicas; as duas primeiras consultadas aceitaram participar.

A coleta de dados com cada família ocorreu na própria residência e foi realizada em dois encontros, conforme a disponibilidade dos participantes. No primeiro dia de coleta, os genitores foram entrevistados, individualmente, seguindo as orientações do RE-HSE-P. As entrevistas foram gravadas em áudio e posteriormente transcritas. No segundo encontro, foi realizada a observação da interação em situação de brincadeira estruturada (aplicação do jogo de memória), primeiramente entre mãe e criança, seguida da observação da interação pai-criança. O jogo de memória foi escolhido por ser muito conhecido e apropriado para a idade das crianças pesquisadas. Ele foi utilizado em um estudo piloto para verificar: a) a familiaridade dos participantes com o jogo ou o fácil aprendizado; b) a posição da câmera; c) a possibilidade de observação de ambos os participantes na filmagem; e d) o tempo de duração necessário para a análise da interação, tendo sido, no estudo piloto, utilizado o jogo da memória de 36 e 72 peças, e o segundo foi escolhido porque a brincadeira levava mais tempo para terminar. Como última etapa da coleta, a pesquisadora se reuniu com a 
mãe e depois com o pai e apresentou oralmente a lista de comportamentos do $\mathrm{CBCL}$, registrando as respostas obtidas.

\section{Procedimentos de tratamento e análise de dados}

Os instrumentos de relato (RE-HSE-P, RE-HSE-Pr, CBCL, TRF) foram categorizados conforme orientações próprias dos manuais, de forma a obter classificações de clínico e de não clínico para os comportamentos avaliados, como descrito em Instrumentos (Método), de acordo com as respostas das mães, pais e professoras. Os dados foram lançados em planilhas Excel para posteriormente serem computados.

Quanto à análise das observações, foram descartados os três minutos iniciais para adaptação dos participantes à tarefa e os dois finais, considerando então 10 minutos de filmagem com a mãe e 10 com o pai, totalizando, nas quatro famílias, 80 minutos. 0 protocolo de observação continha as categorias e subcategorias descritas em Instrumentos (Método), que foram lançados em planilhas Excel para serem computados na sequência. Os comportamentos dos adultos e das crianças, ocorridos na observação, foram registrados minuto a minuto. Todas as observações foram analisadas e categorizadas por dois observadores independentes, totalizando $85 \%$ de acordo.

\section{Resultados e discussão}

A seção de resultados apresenta os dados de relatos e de observação, considerando os diferentes instrumentos e respondentes para problemas de comportamento, habilidades sociais infantis, variáveis de contexto e práticas educativas. Na Tabela 1, encontram-se os dados sobre problemas de comportamento das quatro crianças avaliadas, considerando os diferentes instrumentos de avaliação: a observação e os instrumentos de relato (RE-HSE-P, RE-HSE-Pr, CBCL, TRF) dos três diferentes respondentes (pais, mães e professoras). As 
crianças André e Beatriz foram avaliadas pelas professoras, por meio do TRF, como sem problemas de comportamento, e Caio e Daiane, como apresentando-os.

Tabela 1. Descrição dos comportamentos das crianças (problemas de comportamento) segundo diferentes informantes (pais, mães e professoras) a partir dos instrumentos de relato e da observação da interação mães-crianças e pais-crianças

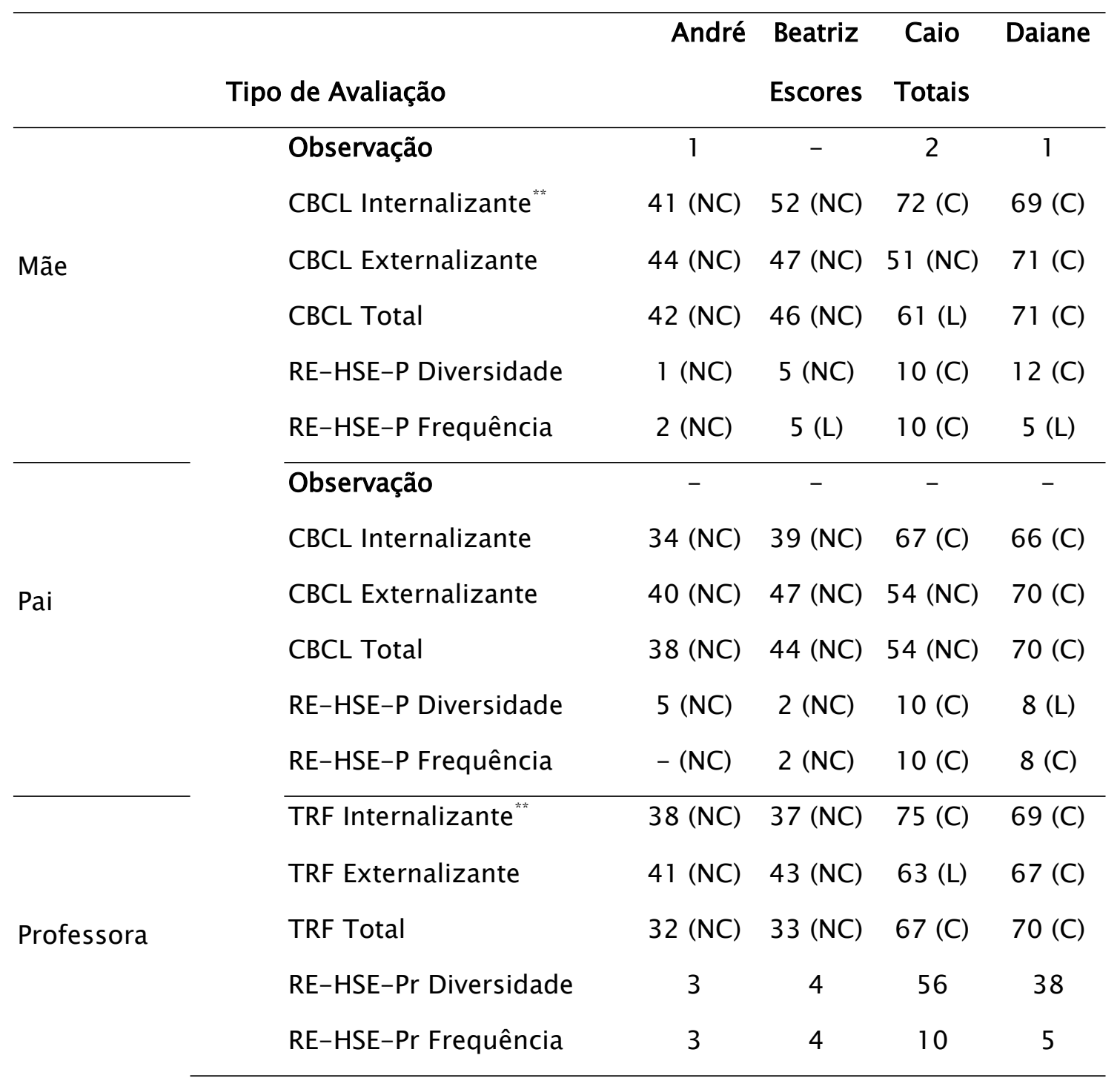

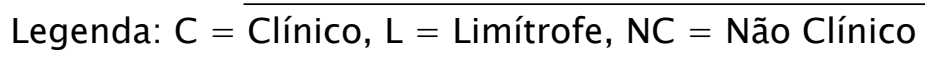

Os escores das professoras não podem ser classificados em clínico e não clínico. Instrumento (RE-HSE-Pr) em processo de validação.

"* Percentis obtidos com os instrumentos CBCL e TRF.

Fonte: Elaborada pelas autoras. 
De acordo com a Tabela 1, nota-se que as indicações das professoras, através do TRF, sobre as crianças que apresentavam ou não problemas de comportamento, foram confirmadas pelas avaliações de mães e pais, seja no CBCL, seja no RE-HSE-P. Então, os comportamentos das crianças foram julgados como não clínicos (André e Beatriz) ou clínicos (Caio e Daiane), nos dois ambientes, escola e família, evidenciando uma maior complexidade de problemas no caso de Caio e Daiane. Interessante destacar que, na situação de observação, os problemas de comportamento das crianças não ocorreram ou foram praticamente inexistentes, indicando que a qualidade da interação genitores-filhos interfere nos comportamentos das crianças, pois em situações lúdicas de jogo, as mães, pais e as crianças foram habilidosas, o que está de acordo com achados da literatura (Bolsoni-Silva et al., 2016; Borden et al., 2014; Marin et al., 2012), que verificou que, quanto mais presentes as práticas educativas positivas, também o são as habilidades sociais infantis.

No entanto, no ambiente escolar, a partir do RE-HSE-Pr, a professora indicou grande diversidade de problemas de comportamento para Caio e Daiane, sendo maior que os apontados por pais e mães dessas crianças, concordando que as crianças respondem a contextos diferentes e que as avaliações de pais e professores podem divergir (Korsch \& Petermann, 2013; Lavigne et al., 2014; Rescorla et al., 2014; Rudasill et al., 2014). Crianças consideradas com problemas de comportamento, tanto pela professora como pela mãe, apresentam mais dificuldade em lidar com os desafios interpessoais do ambiente escolar, mais complexo e exigente, do que o ambiente de sua casa, concordando com Bolsoni-Silva et al. (2006). Como consequência, seus problemas intensificam-se na escola, o que explica a avaliação aparentemente mais severa da professora em relação à dos genitores, nesses casos. A escola exige das crianças comportamentos nem sempre cobrados na família, como ter que ficar muito tempo sentado, dividir brinquedos e materiais com muitas crianças, o que pode explicar maior frequência de problemas de comportamento no ambiente coletivo, justificando o estudo das interações nos dois contextos, como já indicado pela literatura 
(Bernedo et al., 2014; Ercan et al., 2014; Korsch \& Petermann, 2013; Lavigne et al., 2014; Rescorla et al., 2014; Rudasill et al., 2014).

$\mathrm{Na}$ Tabela 2, encontram-se os achados sobre as habilidades sociais das quatro crianças avaliadas, considerando os diferentes instrumentos de avaliação: a observação e os instrumentos de relato (RE-HSE-P, RE-HSE-Pr, CBCL, TRF) dos três diferentes respondentes (pais, mães e professoras).

Tabela 2. Descrição das habilidades sociais das crianças segundo diferentes informantes (pai, mãe e professora) a partir dos instrumentos de relato e da observação

\begin{tabular}{llcccc}
\hline & & André & Beatriz & Caio & Daiane \\
& Tipo de Avaliação & & Escores & Totais & \\
\hline \multirow{2}{*}{ Mãe } & Observação & 109 & 109 & 55 & 9 \\
& RE-HSE-P Diversidade & $30(\mathrm{NC})$ & $16(\mathrm{NC})$ & $23(\mathrm{NC})$ & $17(\mathrm{NC})$ \\
& RE-HSE-P Frequência & $14(\mathrm{NC})$ & $12(\mathrm{~L})$ & $16(\mathrm{NC})$ & $8(\mathrm{C})$ \\
\cline { 3 - 6 } & Observação & 79 & 94 & 60 & 9 \\
& RE-HSE-P Diversidade & $2(\mathrm{C})$ & $11(\mathrm{NC})$ & $11(\mathrm{NC})$ & $12(\mathrm{NC})$ \\
\cline { 3 - 6 } & RE-HSE-P Frequência & $7(\mathrm{C})$ & $8(\mathrm{C})$ & $6(\mathrm{C})$ & $8(\mathrm{C})$ \\
\cline { 3 - 6 } & RE-HSE-Pr Diversidade & 20 & 48 & 10 & 19 \\
Professora & RE-HSE-Pr Frequência & 8 & 12 & 12 & 6 \\
\cline { 2 - 6 } & & & & &
\end{tabular}

Legenda: $\mathrm{C}=$ Clínico, $\mathrm{L}=$ Limítrofe, NC = Não Clínico

Os escores das professoras não podem ser classificados em clínico e não clínico. Instrumento (RE-HSE-Pr) em processo de validação.

Fonte: Elaborada pelas autoras.

Os achados revelam que todas as crianças apresentaram habilidades sociais, embora na situação de observação, a habilidade social das crianças julgadas não clínicas, na interação com suas mães, foi aproximadamente o dobro das crianças avaliadas como clínicas, concordando, então, em parte, com os estudos que apontaram a relação inversa entre habilidades sociais e problemas de comportamento (Barreto et al., 2011; Bolsoni-Silva 
\& Loureiro, 2011). Essa constatação remete à importância de se utilizar múltiplos instrumentos para avaliar repertórios (Del Prette \& Del Prette, 2006).

Tanto as crianças que obtiveram classificações não clínicas quanto as clínicas apresentaram escores avaliados como clínicos, em habilidades sociais, por pelo menos um dos genitores. Uma explicação para esses achados é que o problema de comportamento é multideterminado e o repertório de habilidades sociais é apenas uma das variáveis preditivas (Lins et al., 2012). No entanto, pode ser que as crianças, julgadas como apresentando comportamento clínico, emitam as respostas de habilidades sociais, as quais não produzem atenção suficiente na interação com os interlocutores, dificultando a resolução de problemas. A literatura oferece esse respaldo, por exemplo, no estudo de Leme e BolsoniSilva (2010), que verificou que as crianças categorizadas como clínicas para problemas de comportamento, quando eram habilidosas, no geral eram ignoradas pelas mães ou até mesmo punidas. Bolsoni-Silva e Mariano (2014), ao compararem as interações estabelecidas entre professora e alunos avaliados como clínicos e não clínicos, notaram que as professoras valorizavam os comportamentos de habilidades sociais nas crianças categorizadas como do grupo não clínico, ou seja, que não apresentavam problemas de comportamento, o que não ocorria com as do grupo julgado clínico. As crianças André e Beatriz, que não foram classificadas como apresentando problemas de comportamento, mostraram deficit em habilidades sociais também, indicando que a promoção de competência social, no sentido da prevenção universal, é importante e deveria ser implementada na escola e nas famílias (World Health Organization [WHO], 2004). Destaca-se a complementaridade entre a coleta por meio do relato e da observação, concordando com Del Prette e Del Prette (2006), que trabalham com habilidades sociais e afirmam a importância de utilizar avaliação multimodal. Considerar na coleta de dados diferentes respondentes e instrumentos para avaliar os mesmos construtos torna-se importante porque, em cada ambiente e na interação com diferentes interlocutores, a criança pode responder de maneira distinta. E, quanto aos instrumentos, a utilização de relato e observação é relevante, pois, cada um deles, tem potencialidades e limitações. Um número 
maior de ocorrência de comportamentos de habilidades sociais apareceu na observação, o que sugere que os respondentes, ainda que identifiquem os comportamentos habilidosos das crianças, talvez não os identifiquem e valorizem em sua totalidade. Outro ponto é que na observação tratava-se de uma atividade lúdica, que pode ter facilitado a ocorrência de respostas como "fala coisas engraçadas", "muda comportamentos conforme solicitado", "ouve o que adultos têm a dizer", diferentemente dos roteiros de entrevista que investigam práticas educativas e como as crianças se comportam em diversas situações, além das lúdicas. Os escores obtidos com as professoras, a partir do relato, sugerem padrão semelhante ao dos genitores, como já verificado em Bolsoni-Silva e Mariano (2014), indicando que as interações professor-aluno, ainda que tenham peculiaridades, apresentam características similares às estabelecidas nas famílias, no que refere a manejar o comportamento infantil.

A Tabela 3 apresenta os escores obtidos com as práticas positivas (HSE-P, HSE-PR e contexto) e práticas educativas negativas. 
Tabela 3. Descrição dos comportamentos dos informantes (pai, mãe, professora) quanto às práticas positivas de educação (habilidades sociais educativas - HSE e práticas negativas de educação com cada uma das crianças, a partir de relato e de observação

\begin{tabular}{|c|c|c|c|c|c|c|}
\hline \multicolumn{3}{|c|}{ (RE-HSE-P e RE-HSE-Pr) - Escores Totais } & \multirow{2}{*}{$\begin{array}{c}\text { André } \\
57\end{array}$} & \multirow{2}{*}{$\begin{array}{c}\text { Beatriz } \\
68\end{array}$} & \multirow{2}{*}{$\begin{array}{c}\text { Caio } \\
66\end{array}$} & \multirow{2}{*}{$\begin{array}{c}\text { Daiane } \\
91\end{array}$} \\
\hline & Habilidades & Observação & & & & \\
\hline & Sociais & Diversidade & $3(C)$ & $6(C)$ & $5(C)$ & $7(\mathrm{C})$ \\
\hline \multirow[t]{7}{*}{ Pai } & Educativas & Frequência & $7(C)$ & $8(C)$ & $6(\mathrm{C})$ & $5(C)$ \\
\hline & (HSE-P) & & & & & \\
\hline & Práticas & Observação & - & - & - & - \\
\hline & Educativas & Diversidade & $4(\mathrm{NC})$ & $4(\mathrm{NC})$ & $7(\mathrm{C})$ & $6(\mathrm{~L})$ \\
\hline & Negativas & Frequência & $7(\mathrm{NC})$ & $4(\mathrm{NC})$ & $7(\mathrm{NC})$ & $6(\mathrm{NC})$ \\
\hline & Habilidades & Observação & 117 & 106 & 59 & 15 \\
\hline & Sociais & Diversidade & $9(\mathrm{~L})$ & $11(\mathrm{NC})$ & $8(\mathrm{~L})$ & $7(\mathrm{C})$ \\
\hline \multirow[t]{7}{*}{ Mãe } & Educativas & Frequência & $8(C)$ & $10(\mathrm{C})$ & $12(\mathrm{NC})$ & $5(C)$ \\
\hline & (HSE-P) & & & & & \\
\hline & Práticas & Observação & - & - & - & - \\
\hline & Educativas & Diversidade & $2(\mathrm{NC})$ & $4(\mathrm{NC})$ & $7(\mathrm{C})$ & 8 (C) \\
\hline & Negativas & Frequência & $2(\mathrm{NC})$ & $4(\mathrm{NC})$ & $7(C)$ & $8(\mathrm{NC})$ \\
\hline & Habilidades & Diversidade & 15 & 34 & 29 & 42 \\
\hline & Sociais & Frequência & 11 & 14 & 20 & 18 \\
\hline \multirow[t]{5}{*}{ Professora } & Educativas & & & & & \\
\hline & (HSE-Pr) & & & & & \\
\hline & Práticas & Diversidade & 1 & 2 & 8 & 5 \\
\hline & Educativas & Frequência & 1 & 4 & 8 & 3 \\
\hline & Negativas & & & & & \\
\hline
\end{tabular}

Fonte: Elaborada pelas autoras.

Conforme a Tabela 3, parece que as práticas negativas dos genitores se apresentam em maior frequência para as crianças avaliadas como clínicas do que para as julgadas não 
clínicas, pois, tanto para Caio como para Daiane, pais e mães responderam utilizar práticas negativas com diversidade e/ou frequência com classificação limítrofe ou clínica, o que não ocorreu para André e Beatriz (Alvarenga et al., 2012; Borden et al., 2014; Bolsoni-Silva \& Loureiro, 2011; Shaffer et al., 2013; Xing \& Wang, 2013). Esse dado vem ao encontro da literatura, ou seja, crianças sem problemas de comportamento são pouco punidas porque emitem menos comportamentos que os genitores desaprovam (Bolsoni-Silva \& Mariano, 2014; Bolsoni-Silva \& Loureiro, 2011; Borden et al., 2014; Bueno et al., 2011, O'Connor et al., 2011; Silver et al., 2010), e os adultos, que os educam, apresentam mais maneiras habilidosas no estabelecimento de limites (Leme e Bolsoni-Silva, 2010; Marin et al., 2012). As respostas das professoras também apresentaram o mesmo padrão dos pais, tal como já destacado por Bolsoni-Silva e Mariano (2014).

Como já apontado na Tabela 2, há deficit de habilidades sociais educativas também no grupo não clínico, pois se observa escores clínicos para as práticas positivas (HSE-P e contexto) para todas as crianças avaliadas, ao menos por um dos genitores. No que se refere aos dados de observação, nota-se que, para os pais, os escores são próximos. No entanto, para as mães, verifica-se muita diferença, sendo altos escores para as interações com as crianças avaliadas como não apresentando problemas de comportamento (não clínicas), em detrimento das que o apresentam (clínicas), sobretudo para Daiane, a qual apresenta mais comprometimento quanto a problemas de comportamento (Tabela 1), apontando para as diferenças existentes entre as interações estabelecidas com as mães e com os pais, sendo as primeiras, geralmente, mais presentes e habilidosas (Bolsoni-Silva \&Marturano, 2008).

Os resultados vão em direção de estudos que constatam que a alta ocorrência de habilidades sociais corresponde à alta frequência de práticas positivas (Bolsoni-Silva et al., 2016; Borden et al., 2014; Marin et al., 2012), bem como entre práticas negativas e problemas de comportamento (Alvarenga et al., 2012; Borden et al., 2014; Bolsoni-Silva \& Loureiro, 2011; Shaffer et al., 2013; Xing \& Wang, 2013). 
A Tabela 4 apresenta a frequência dos comportamentos de pais e de mães, considerando tanto o relato (RE-HSE-P e CBCL) quanto a análise da situação de observação por meio das mesmas categorias.

Tabela 4. Ocorrência de comportamentos dos pais e das mães quanto às categorias específicas de diversidade das práticas educativas positivas (habilidades sociais educativas), práticas negativas, problemas de comportamento e habilidades sociais infantis, a partir de relato - RE-HSE-P e da observação da situação de jogo de memória

\begin{tabular}{lcccccccccc}
\hline & \multicolumn{2}{c}{ André } & \multicolumn{2}{c}{ Beatriz } & \multicolumn{2}{c}{ Caio } & \multicolumn{2}{c}{ Daiane } \\
\cline { 2 - 11 } & Rel. & Obs. & Rel. & Obs. & Rel. & Obs. & Rel. & Obs. \\
& M P & M P & M P & M P & M P & M P & M P & M P \\
\hline HSE-P Comunicação e & 41 & 5126 & 62 & 4029 & 23 & 1521 & 34 & 743
\end{tabular}

negociação

\begin{tabular}{llllllllll}
\hline HSE-P Expressão & 52 & 6631 & 54 & 6639 & 62 & 4445 & 43 & 848
\end{tabular}

Sentimentos

Enfrentamento

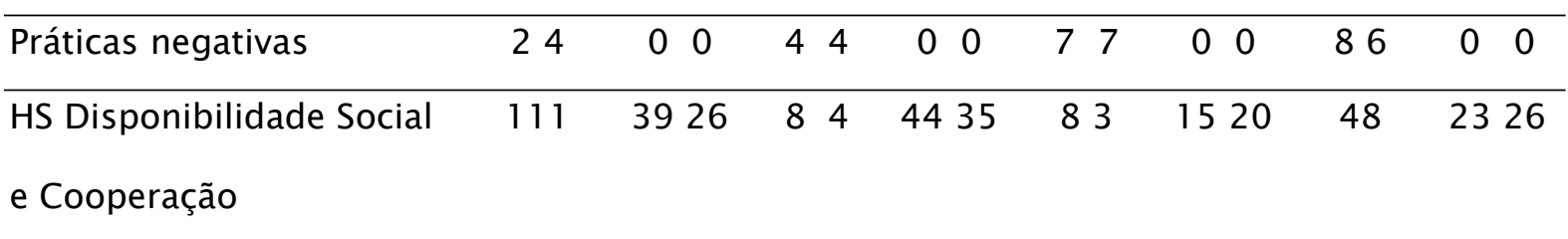

\begin{tabular}{lllllllllll}
\hline HS Expressão Sentimentos & 191 & 70 & 53 & 4 & 4 & 6559 & 158 & 4041 & 98 & 2861
\end{tabular}

Enfrentamento

\begin{tabular}{lllllllllllllllll}
\hline Problemas de & 0 & 5 & 1 & 0 & 4 & 2 & 0 & 0 & 8 & 5 & 2 & 0 & 8 & 6 & 0 & 0
\end{tabular}

Comportamento

Externalizantes

Problemas de

$\begin{array}{lllllllllllllllll}1 & 0 & 0 & 0 & 1 & 0 & 0 & 0 & 2 & 5 & 0 & 0 & 4 & 2 & 1 & 0\end{array}$

Comportamento

Internalizantes

Legenda: Rel. = Relato, Obs. = Observação, $\mathrm{M}=\mathrm{Mãe}, \mathrm{P}=$ Pai, HSE-P = Habilidades sociais educativas parentais, $\mathrm{HS}=$ Habilidades sociais infantis,

Fonte: Elaborada pelas autoras. 
A Tabela 4 indica a maior frequência dos comportamentos dos pais, tanto de Comunicação e Negociação quanto de Expressão de Sentimentos e Enfrentamento, na situação de observação do que nos relatos por meio dos instrumentos utilizados. Isso pode ter ocorrido devido ao fato de que na situação observacional a interação era lúdica, e nas entrevistas com os instrumentos pergunta-se sobre diferentes interações, incluindo aquelas que envolvem o estabelecimento de limites, por exemplo: dizer não com explicação, estabelecer regras, pedir mudança de comportamento, sendo os comportamentos apontados como importantes para serem avaliados, pois têm forte relação com a ocorrência de problemas de comportamento (Bolsoni-Silva \& Mariano, 2014; Bolsoni-Silva \& Loureiro, 2011 ; Borden et al., 2014; Bueno et al., 2011; Gomide, 2006; Price et al., 2013; Salvo et al., 2005; Silver et al., 2010; Trepat et al., 2014; Xing \& Wang, 2013).

As mães das crianças julgadas não clínicas parecem ser mais habilidosas do que as do grupo clínico na situação de observação. No entanto, o pai de Daiane destaca-se na interação com sua filha, indicando um bom relacionamento, o que não ocorre com sua esposa, que provavelmente é quem mais fica com a criança nas interações cotidianas.

Pela Tabela 4, também nota-se um número grande de ocorrências para os comportamentos de disponibilidade social e cooperação e de expressão de sentimentos e enfrentamento das crianças, concordando com a literatura (por exemplo Bolsoni-Silva \& Loureiro, 2016). A distribuição entre a ocorrência de comportamentos a partir dos pais e das mães variou bastante; no entanto, a partir do relato, as mães das crianças avaliadas como apresentando comportamento não clínico e clínico, geralmente, foram capazes de identificar um número maior de comportamentos de habilidades sociais do que os pais, confirmando pesquisa prévia (Bolsoni-Silva \& Marturano, 2008). Quanto aos dados da observação para as crianças que obtiveram escores não clínicos, houve maior ocorrência de habilidades sociais na presença da mãe do que do pai; já para as julgadas como clínicas, o número de ocorrência de habilidades sociais foi maior na interação com os pais, o que pode refletir que os pais brincam mais com as crianças no fim de semana e estabelecem menos 
limites (Bolsoni-Silva \& Marturano, 2008). Destaca-se que as crianças já apresentavam comportamentos de habilidades sociais, na avaliação via relato e observação para as crianças com e sem problemas de comportamento, e se os genitores aprenderem a valorizá-los em diferentes situações, possivelmente haverá redução dos problemas de comportamento (Bolsoni-Silva et al., 2016; Borden et al., 2014; Marin et al., 2012). Intervenções com os genitores devem estimular situações de brincadeiras, nas quais as crianças têm oportunidade de serem habilidosas e seus pais e mães também, melhorando a interação entre eles e, portanto, ampliando a competência social e reduzindo os problemas.

De acordo com a Tabela 4, nota-se baixa ocorrência de problemas de comportamento, seja a partir do relato verbal (RE-HSE-P), seja da observação, especialmente para as crianças avaliadas como não clínicas. Os resultados reafirmam os dados descritos na Tabela 1. Importante destacar que mesmo as crianças avaliadas como não clínicas emitiram problemas de comportamento externalizantes e internalizantes (Bolsoni-Silva et al., 2016). Chama a atenção que mesmo as crianças com indicadores de problemas de comportamento, em situação lúdica, foram habilidosas, reiterando que se os pais conseguissem criar condições para ocorrer interações positivas os comportamentos habilidosos dos filhos poderiam ser fortalecidos (Bolsoni-Silva et al., 2016).

Já os problemas de comportamento ocorreram com baixa frequência na situação de observação, mas foram bem relatados pelos CBCL, TRF, RE-HSE-P, RE-HSE-Pr, em diversas situações, nas quais possivelmente os pais e professoras usam de práticas negativas de educação, especialmente para as crianças com indicadores de problemas de comportamento, o que é respaldado por diversos estudos (Price et al., 2013; Trepat et al., 2014; Xing \&Wang, 2013). As queixas de problemas externalizantes são as mais frequentes por pais e professores (Bolsoni-Silva et al., 2015; Pesce, 2009) e foi confirmado no presente estudo. De maneira geral, os resultados da presente pesquisa reafirmam que práticas positivas que incluem afeto (Bolsoni-Silva \& Loureiro, 2011; O’Connor et al., 2011 ; Silver et al., 2010), comunicação (Bolsoni-Silva \& Mariano, 2014; Borden et al., 2014; Bueno et al., 2011 ) e estabelecimento de limites com consistência (Leme \& Bolsoni-Silva, 2010; Marin et 
al., 2012), indicando a baixa frequência de problemas de comportamento e alta frequência de habilidades sociais. E, ao contrário, práticas negativas estão relacionadas a problemas de comportamento (Alvarenga et al., 2012; Borden et al., 2014; Bolsoni-Silva \& Loureiro, 2011 ; Shaffer et al., 2013; Xing \& Wang, 2013). A literatura tem encontrado tais relações nas interações pais e filhos - com a presente pesquisa notam-se interações sociais semelhantes, no que se refere à interação professor-aluno (Bolsoni-Silva \& Mariano, 2014).

\section{Considerações finais}

Este estudo exploratório descreveu o repertório comportamental infantil de crianças consideradas como pertencendo ao grupo clínico e não clínico para problemas de comportamento relatado por diferentes interlocutores - mães, pais e professoras - em ambientes distintos, família e escola, utilizando vários instrumentos de coleta de dados e a situação de observação lúdica entre pais-crianças e mães-crianças. Consideramos como ponto forte desta pesquisa os múltiplos interlocutores, de dois ambientes diferentes escola e família - e a situação de observação utilizando as mesmas categorias dos instrumentos de relatos de pais, mães e professoras. Como limitação, sem dúvida, concluímos que o pequeno número de participantes foi a principal.

Embora com número reduzido de participantes, espera-se que esta pesquisa possa ser inspiradora de outras e que o conhecimento sobre práticas educativas, habilidades sociais e problemas de comportamento em diferentes contextos possa ser mais bem explorado e utilizado na prática educativa e clínica. Futuros estudos podem ser conduzidos com amostra maior e com outros recursos de observação das interações pais-filhos e professor-aluno, incluindo situações de tarefa escolar, além de situação de jogo. 


\section{Referências}

Achenbach, T. M., \&Rescorla, L. A. (2001). Manual for the ASEBA School-Age Forms \& Profiles. Burlington, VT: University of Vermont, Research Center for Children, Youth \& Families.

Alvarenga, P., Magalhães, M. O., \& Gomes, Q. S. (2012). Relações entre práticas educativas maternas e problemas de externalização em pré-escolares. Estudos de Psicologia, $29,33-42$.

Barreto, S. O., Freitas. L. C., \& Del Prette, Z. A. P. (2011). Habilidades sociais na comorbidade entre dificuldades de aprendizagem e problemas de comportamento: uma avaliação multimodal. Psico, 42, 503-510.

Bernedo, I. M., Salas, M. D., Fuentes, M. J., \& García-Martin, M. A. (2014). Foster Children's Behavior Problems and Impulsivity in the Family and School Context. Children and Youth Services Review, 42, 43-49.

Bolsoni-Silva, A. T., \& Loureiro, S. R. (2016). Simultaneous Assessment of Social Skills and Behavior Problems: Education and Gender. Estudos de Psicologia (Campinas), 33, 453-464.

Bolsoni-Silva, A. T., Levatti, G. E., Guidugli, P. M., \& Marim, V. C. C. (2015). Problemas de comportamento, em ambiente familiar em escolares e pré-escolares diferenciados pelo sexo. Revista Interamericana de Psicologia/Interamerican Journal of Psychology, $49,354-364$.

Bolsoni-Silva, A. T., Loureiro, S., \& Marturano, E. M. (2016). Roteiro de entrevista de habilidades sociais educativas parentais (RE-HSE-P). Manual Técnico. São Paulo: Hogrefe. 
Bolsoni-Silva, A. T., Marturano, E. M., \& Loureiro, S. (2018). Roteiro de entrevista de habilidades sociais educativas para professores (RE-HSE-Pr). Manual Técnico. São Paulo: Hogrefe.

Bolsoni-Silva, A. T., \& Mariano, M. L. (2014). Práticas educativas de professores e comportamentos infantis, na transição ao primeiro ano do Ensino Fundamental. Estudos e Pesquisas em Psicologia (Online), 14, 814-833.

Bolsoni-Silva, A. T., Loureiro, S. R. (2011). Práticas educativas parentais e repertório comportamental infantil: comparando crianças diferenciadas pelo comportamento. Paidéia, 21, 61-71.

Bolsoni-Silva, A. T., Marturano, E. M., Figueiredo, V. A. P., \& Manfrinato, J. W. S. (2006). Habilidades sociais e problemas de comportamento de pré-escolares: comparando avaliações de mães e de professoras. Psicologia: Reflexão e Crítica, 19, 460-469.

Bolsoni-Silva, A. T., Marturano, E. M. (2008). Habilidades sociais educativas parentais e problemas de comportamento: comparando pais e mães de pré-escolares. Aletheia (ULBRA), 27, 126-138.

Borden, L. A., Herman, K. C., Stormont, M., Nidhi-Goel, N., Darney, D., Reinke, W. M., \& Webster-Stratton, C. (2014). Latent Profile Analysis of Observed Parenting Behaviors in a Clinic Sample. Journal Abnormal Child Psychology, 42, 731-742.

Bordin, I. A., Rocha, M. M., Paula, C. S., Teixeira, M. C. T., Achenbach, T. M., Rescorla, L. A., \& Silvares, E. F. (2013). Child Behavior Checklist (CBCL), Youth Self-Report (YSR) and Teacher's Report Form (TRF): an Overview of the Development of the Original and Brazilian Versions. Cadernos de Saúde Pública, 29, 13-28.

Bueno, A. C. W., Grossi, R., Santo, B. C., Silva, L. C., \& Moura, C. B. (2011). Comparação entre comportamentos apresentados por mães de pré-escolares clínicos e não clínicos em 
uma situação lúdica. Revista Brasileira de Terapia Comportamental e Cognitiva, 13, $4-20$.

Casali-Robalinho, I. G., Del Prette, Z. A. P., \& Del Prette, A. (2015). Social Skills as Predictors of Problem Behavior in School Children. Psicologia: Teoria e Pesquisa, 31, 321-330.

Del Prette, Z. A. P., \& Del Prette, A. (2006). Psicologia das habilidades sociais na infância. Petrópolis: Vozes.

Dessen, M. A. (2009). Questionário de caracterização do sistema familiar: Versão - pais ou responsável. In L. Weber \& M. A. Dessen (Org.). Pesquisando a família: instrumentos para coleta e análise de dados (pp.115-131). Curitiba, PR: Juruá.

Ercan, E. S., Bilaç, Ö., Özaslan, T. U., \& Rohde, L. A. (2014). Is the Prevalence of ADHD in Turkish Elementary School Children Really High?. Social Psychiatry and Psychiatric Epidemiology, 50, 1145-1152.

Gomide. P. I. C. (2006). Inventário de Estilos Parentais. Modelo Teórico: Manual de Aplicação, apuração e interpretação. Petrópolis: Vozes.

Korsch, F., \& Petermann, F. (2013). Agreement between Parents and Teachers on Preschool Children's behavior in a Clinical Sample with Externalizing Behavioral Problems. Child Psychiatry Human Development, 45, 617-627.

Lavigne, J. V., Dahl, K. P., Gouze, K. R., Lebailly, S. A., \& Hopkins, J. (2014). Multi-Domain Predictors of Oppositional Defiant Disorder Symptoms in Preschool Children: CrossInformant Differences. Child Psychiatry Human Development, 46, 308-319.

Leme, V. B. R., \& Bolsoni-Silva, A. T. (2010). Habilidades sociais educativas parentais e comportamentos de pré-escolares. Estudos de Psicologia (UFRN), 15, 161-173. 
Lins, T., \& Alvarenga, P. (2015). Controle psicológico materno e problemas internalizantes em pré-escolares. Psicologia: Teoria e Pesquisa, 31, 311-319.

Lins, T. C. S., Alvarenga, P., Santos, C. P., Almeida, P., \& Santos, H. C. (2012). Problemas externalizantes e agressividade infantil: uma revisão de estudos brasileiros. Arquivos Brasileiros de Psicologia, 64, 57-75.

Maia, R. C. C., Soares, A. B., \& Victoria, M. S. (2010). Um estudo com professores da educação infantil e do ensino fundamental sobre suas habilidades sociais e inteligência geral. Estudos e Pesquisas em Psicologia, 9, 464-479.

Mariano, M. (2015). Práticas educativas de professores, habilidades sociais e problemas de comportamento: um estudo comparativo, correlacional e preditivo. Dissertação de mestrado, Curso de Pós-Graduação em Psicologia do Desenvolvimento e Aprendizagem, Universidade Estadual Paulista, Unesp, Bauru, São Paulo, Brasil.

Mariano, M. L. (2011). Análise de práticas educativas de professores na interação com alunos com e sem problemas de comportamento. Dissertação de mestrado não publicada, Curso de Pós-Graduação em Psicologia do Desenvolvimento e Aprendizagem, Universidade Estadual Paulista, Unesp, Bauru, São Paulo, Brasil.

Marin, A. H., Piccinini, C. A., Gonçalves, T. R., \& Tudge, J. R. H. (2012). Práticas educativas parentais, problemas de comportamento e competência social de crianças em idade pré-escolar. Estudos de Psicologia, 17, 5-13.

Merrell, K. W., \& Harlacher, J. E. (2008). Behavior Rating Scales. In R. P. Archer \& S. R. Smith (Eds.). Personality Assessment (pp. 247-280). New York, Routledge/Taylor \& Francis.

O'Connor, E. E., Dearing, E., \& Collins, B. A. (2011). Teacher-Child Relationship and Behavior Problem Trajectories in Elementary School. American Educational Research Journal, 48, 120-162. 
Patterson, G., Reid, J., \& Dishion, T. (2002). Antisocial boys. Comportamento antissocial. Santo André: ESETec.

Pesce, R. (2009). Violência familiar e comportamento agressivo e transgressor na infância: uma revisão da literatura. Ciência e Saúde Coletiva, 14, 507-518.

Price, J. M., Chiapa, A., \& Walsh, N. E. (2013). Predictors of Externalizing Behavior Problems in Early Elementary-Aged Children: the Role of Family and Home Environments. The Journal of Genetic Psychology: Research and Theory on Human Development, 174, 464-471.

Reis, V. T. C., Prata, M. A. R., \& Soares, A. B. (2012). Habilidades sociais e afetividade no contexto escolar: perspectivas envolvendo professores e ensino-aprendizagem. Psicologia Argumento, 30, 347-357.

Rescorla, L. A., Bochicchio, L., Achenbach, T. M., Ivanova, M. Y., Almqvist, F., Begovac, I., \& Verhulst, F. C. (2014). Parent-Teacher Agreement on Children's Problems in 21 societies. Journal of Clinical Child \& Adolescent Psychology, 43, 627-642.

Rudasill, K. M., Prokasky, A., Tu, X., Frohn, S., Sirota, K., \& Molfese, V. J. (2014). Parent vs. Teacher Ratings of Children's Shyness as Predictors of Language and Attention Skills. Learning and Individual Differences, 34, 57-62.

Sabbag, G. M., \& Bolsoni-Silva, A. T. (2015). Relações entre as práticas educativas e as habilidades sociais educativas maternas em adolescentes. In Z. A. P. Del Prette, A. B. Soares, C. S. Pereira-Guizzo, M. F. Wagner \& V. B. R., Leme (Orgs.). Habilidades sociais: diálogos e intercâmbios sobre pesquisa e prática (pp. 102-127). Novo Hamburgo: Sinopsys.

Salvo, C. G., Silvares, E. F. M., \& Toni, P. M. (2005). Práticas educativas como forma de predição de problemas de comportamento e competência social. Estudos de Psicologia, 22, 187-195. 
Shaffer, A., Lindhiem, O., Kolko, D., \& Trentacosta, C. (2013). Bidirectional Relations between Parenting Practices and Child Externalizing Behavior: a Cross-Lagged Panel Analysis in the Context of a Psychosocial Treatment and 3-year Follow-Up. Journal of Abnormal Child Psychology, 41, 199-210.

Silver, R. B., Measelle, J. R., Armstrong, J. M., \& Essex, M. J. (2010). The Impact of Parents, Child Care Providers, Teachers, and Peers on Early Externalizing Trajectories. Journal of School Psychology, 48, 555-583.

Soares, A. B., \& Mello, T. V. S. (2010). Habilidades sociais entre professores e não professores. Revista Brasileira de Terapias Cognitivas, 5, 15-27.

Trepat, E., Granero, R., \& Ezpeleta, L. (2014). Parenting Practices as Mediating Variables between Parents' Psychopathology and Oppositional Defiant Disorder in Preschoolers. Psicothema, 26, 497-504.

World Health Organization - WHO. (2004). Prevention of Mental Disorders - Effective Interventions and Policy Options. Paris: França. Retirado de http://www.who.int/mental_health/evidence/en/prevention_of_mental_disorders_sr. pdf.

Xing, X., \& Wang, M. (2013). Sex Differences in the Reciprocal Relationships between Mild and Severe Corporal Punishment and Children's Internalizing Problem Behavior in a Chinese Sample. Journal of Applied Developmental Psychology, 34, 9-16.

Recebido em: 17/4/2018

Aprovado em: $27 / 1 / 2020$ 